\title{
Similitudes y diferencias entre repositorios y redes sociales. Por qué un repositorio no es una red social, ni debe serlo
}

Francisco Martínez Galindo | Servicio de Biblioteca y Documentación Científica, Universitat Politècnica de València

URL de la contribución <www.iaph.es/revistaph/index.php/revistaph/article/view/4670>

ResearchGate, Academia.edu... me ha pedido que suba mis publicaciones, ¿es legal? ¿Cumple la política de acceso abierto de la universidad? ¿Y el mandato del financiador? ¿Cuál es la diferencia entre una red social académica y el repositorio institucional? Todas estas preguntas y más recibimos en los repositorios universitarios de forma habitual. Para responderlas lo mejor es comprender en qué consiste cada servicio y cuál es su lugar en el ecosistema de comunicación actual.

Las redes sociales académicas proporcionan a la comunidad investigadora una experiencia similar a Facebook o LinkedIn al conectar a los investigadores con sus pares. Estas plataformas invitan a crear perfiles con su currículum y subir sus publicaciones estableciendo redes con los investigadores con los que trabajan, coautores o interesados en sus investigaciones y que leen sus obras, las comentan, difunden y citan.

Los repositorios institucionales, generalmente gestionados por bibliotecas, difunden las publicaciones de sus investigadores en "acceso abierto", RiuNet en el caso de la Universitat Politècnica de València (UPV). Su objetivo principal es hacer que los resultados académicos de la universidad estén lo más disponibles posible y asegurar su preservación.

En el caso de los repositorios temáticos, normalmente gestionados por los propios investigadores, recogen las publicaciones en una disciplina particular, facilitando a los autores de un determinado campo compartir y solicitar comentarios sobre su trabajo de colegas en ese ámbito, independientemente de dónde trabajen.

Aunque a primera vista, repositorios y redes sociales comparten objetivos, lo interesante son sus diferencias.
Las redes sociales tienen, generalmente, un objetivo comercial en el que necesitan un rendimiento económico de su inversión y trabajo y, teóricamente, podrían cerrar en cualquier momento. Academia.edu no ha cerrado, pero sí realizó un cambio en su modelo de negocio pasando a cobrar a los propios investigadores por los servicios prémium. Solo si tienen éxito pueden sobrevivir.

Los repositorios institucionales, financiados por la universidad o institución que los alberga, incluyen en su objetivo la preservación a largo plazo, de la misma forma que mantienen en sus compactus las revistas compradas desde hace años para permitir su acceso futuro, independientemente de que las editoriales desaparezcan.

Cuanto más se difunden los artículos mejor para todos, editores, autores, financiadores... Por un lado se consigue que la investigación llegue a otros autores (es la idea de publicar, ¿verdad?); por otro, amplían la posibilidad de aumentar las citas, que es el objetivo real que persiguen los investigadores debido a las presiones del sistema actual. Tanto las redes sociales académicas como los repositorios coinciden en ayudar a los investigadores en esta difusión, pero se observan claras diferencias.

Los derechos de las publicaciones. Lo normal es que se transfieran a los editores, lo que impide a los autores difundir sus trabajos. Sin embargo, con frecuencia los co-autores desconocen si han transferido los derechos o el tipo de licencia firmada, ya que eso lo gestiona el autor de correspondencia, incluso con artículos publicados en acceso abierto.

Los repositorios institucionales se aseguran de cumplir los requerimientos de las editoriales respecto a la difu- 


\section{a debate Repositorios y redes sociales académicas para la transferencia del conocimiento abierto \\ | coordina Remedios Melero Melero}

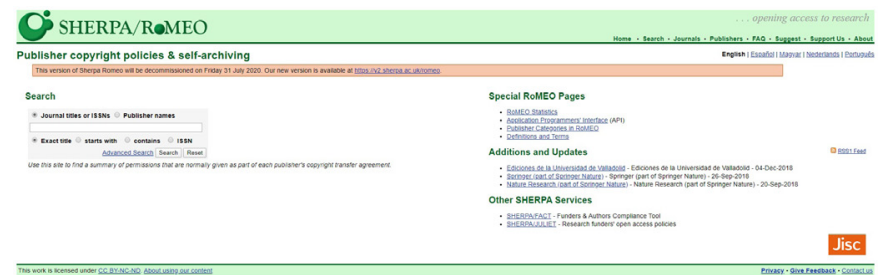

Portal del proyecto Sherpa/Romero

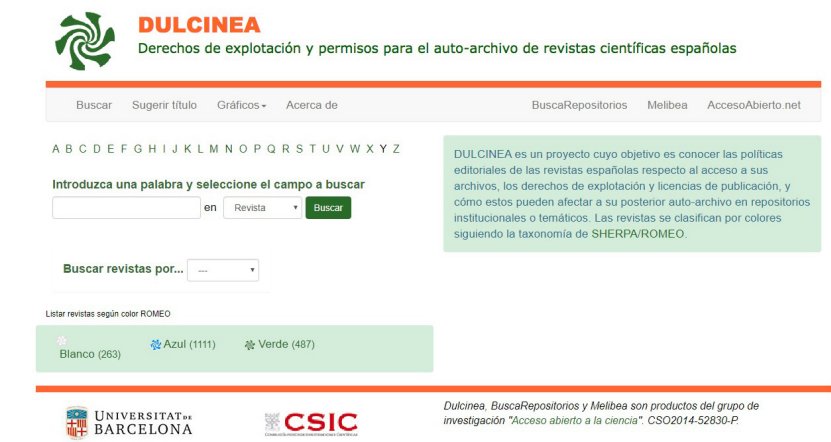

Portal del proyecto Dulcinea

sión en abierto de las publicaciones. La comprobación de qué versión (editorial o postprint) permite la editorial, se realiza comprobando en Sherpa o Dulcinea y, si es necesario, contactando con la editorial. Normalmente se realiza desde el servicio de biblioteca o resolviendo dudas de los autores, en caso de que la política de la institución se decante por el autoarchivo.

En el caso de las redes sociales académicas, para ayudar a la difusión piden, "insistentemente", a sus usuarios que suban el texto completo de sus publicaciones, pero dejan la responsabilidad en el propio investigador, llevando a un tira y afloja con las grandes editoriales. Primero Academia.edu se vio obligada a retirar (ELSEVIER, 2013) las publicaciones de Elsevier. Posteriormente, también Reseachgate ha tenido problemas legales (PUBLISHERS, 2018), viéndose obligada a retirar versiones editoriales que difundía para las que no tenía permisos (COALITION , 2019); recientemente algunas negociaciones (RESEARCHGATE, 2020) parece que han surtido efecto $y$, si consigue aumentar estos acuerdos, puede frenar una desban- dada (CORKER, 2017) de investigadores que prefieran evitar que, después de dedicar su tiempo a rellenar su currículum en cada una de estas plataformas, este sea eliminado.

También el concepto de acceso abierto choca en estas plataformas donde documentos o ficheros están tras el muro que significa tener que identificarse y tampoco facilitan métodos para extraer datos de su sistema. Los repositorios se comprometen con el acceso abierto mediante el uso de estándares internacionales de interoperabilidad, en la descripción de metadatos, normalmente con licencias libres, que favorecen su descripción y recuperación, así como facilitando la comprobación del cumplimiento de los mandatos de las agencias financiadoras por OpenAIRE, Recolecta o cualquier otro buscador académico.

¿Redes sociales académicas o repositorios? Ambos ofrecen algún valor para los investigadores: las primeras permiten encontrar a sus pares y avivan el intercambio con foros de preguntas; también cuentan con sistemas de ranking, a los que tan acostumbrados están, y que recompensan sus participaciones; además no son "rigurosos" con los metadatos y tienen automatizados los procesos de ingesta, siendo los propios repositorios una gran fuente de información. Ante la problemática de la política de las editoriales, es más sencillo depositar el artículo en el repositorio institucional/temático y compartir en la plataforma social la url (handle) de sus contenidos científicos depositados en el repositorio.

Los repositorios de acceso abierto no son sitios de redes sociales pero es interesante que añadan, en la medida de lo posible, funcionalidades -algunas de las cuales se encuentran recogidas en la nueva generación de repositorios (NEXT GENERATION, 2020)- que los académicos encuentran valiosas, como crear un perfil de autor, automatizar la subida de publicaciones, comunicarles los logros conseguidos en visitas y descargas, la comunicación entre distintos CRIS y repositorios -evitando duplicar la introducción en cada aplicación- y permitir alimentarse de las fuentes existentes. 
No debe pasarse por alto el valor proporcionado por el repositorio institucional, particularmente la preservación a largo plazo y el compromiso con el acceso abierto que no ofrecen otros servicios. Si aún no estás utilizando tu repositorio institucional $u$ otro repositorio de acceso abierto, dedica unos minutos para consultar los servicios disponibles.

\section{BIBLIOGRAFÍA}

- COALITION for Responsible Sharing: Status Report on ResearchGate. ResearchGate, junio de 2019 <https:// www.responsiblesharing.org/2019-06-13-status-report-onresearchgate-june-2019/> [Consulta: 12/05/2020]

- CORKER K. (2017) Bye bye, academia.edu and ResearchGate-hello PsyArXiv! Science of psych, 18 de agosto de 2017 <https://scienceofpsych.wordpress. com/2017/08/18/bye-bye-academia-edu-and-researchgatehello-psyarxiv/> [Consulta: 12/05/2020]

- ELSEVIER is taking down papers from Academia.edu (2013) SV-POW! (Sauropod Vertebra Picture of the Week), 6 de diciembre de $2013<$ https://svpow.com/2013/12/06/elsevieris-taking-down-papers-from-academia-edu/> [Consulta: 12/05/2020]

- NEXT GENERATION Repository Working Group (2020) Defining the next generation repository. About the Technologies. COAR Confederation of Open Access Repositories <https:// ngr.coar-repositories.org/technology/> [Consulta: 12/05/2020]

- PUBLISHERS v ResearchGate: round 2 (2018) EASE Blog, 7 de diciembre de 2018. European Association of Science Editors <https://ese-bookshelf.blogspot.com/2018/12/ publishers-v-researchgate-round-2.html?spref=tw> [Consulta: 12/05/2020]

- RESEARCHGATE and Wiley announce cooperation agreement. ResearchGate, 6 de mayo de $2020<$ https://www. researchgate.net/blog/post/researchgate-and-wiley-announcecooperation-agreement> [Consulta: 12/05/2020] 